\title{
Onychophagia and subgingival foreign body embedment: a case report
}

\author{
R. Ellison, ${ }^{1}$ S. Rahman, ${ }^{* 2}$ D. Barker ${ }^{2}$ and C. Larmour ${ }^{3}$
}

IN BRIEF

- Highlights the importance of a thorough clinical assessment in conjunction with a comprehensive and methodical history.

- The reader should give consideration to more unusual sources of infection when forming a differential diagnosis in cases such as the one described.

This paper describes a case of subgingival foreign body embedment in the form of a finger nail fragment, as a consequence of onychophagia (fingernail biting). The result was periodontal infection that was concurrent with apical pathology on an adjacent non-vital tooth. This finding complicated the diagnosis and management of this case. Although foreign body impactions are relatively rare, they should be considered in a differential diagnosis, especially when the clinician is presented with an unusual clinical picture.

\section{BACKGROUND}

There have been few reports of subgingival foreign body embedment in the literature and still fewer describing subgingival fingernail embedment. ${ }^{1}$ Previous reports generally describe periodontal infection secondary to the foreign body. This report describes foreign body embedment resulting in periodontal infection, which was concurrent with apical pathology on an adjacent nonvital tooth, complicating the diagnosis and management. This unusual case emphasises the importance of considering foreign body impaction as part of a differential diagnosis. This is especially relevant in adolescent and paediatric patients and when clinical presentation is unusual and aetiology is uncertain. ${ }^{2}$

\section{CASE REPORT}

A 14-year-old male Caucasian patient was referred for a combined orthodonticrestorative opinion regarding chronic apical pathology affecting his upper left lateral incisor and crowding of his upper arch associated with a buccally displaced upper left canine.

His past medical history was unremarkable apart from reporting a fall three years previously. During this fall he had sustained trauma to his upper left central and lateral

'Dental Practitioner, M\&S Dental Care, Fort William; ${ }^{2}$ Restorative, ${ }^{3}$ Orthodontics, University of Aberdeen Dental School and Hospital

*Correspondence to: Mr Shahab Rahman

Email:Shahab.rahman@nhs.net

\section{Refereed Paper}

Accepted 23 January 2014

DOI: $10.1038 /$ sj.bdj.2014.351

${ }^{\circ}$ British Dental Journal 2014; 216: 519-520 incisors. Following this the fractured central incisor had been restored with composite. The lateral incisor had subsequently become nonvital and had been undergoing root canal therapy with his general dental practitioner over a prolonged period in an attempt to achieve apexification without success.

At his combined consultation appointment he did not report any current symptoms. Extra-oral examination was unremarkable and intra-orally tenderness was elicited apical to the upper left lateral incisor and also around the partially erupted upper left permanent canine, which also had associated gingival inflammation. Both teeth were tender to percussion (Fig. 1). Periodontal probing around the canine revealed an $8 \mathrm{~mm}$ pocket (Fig. 2) on the mesial aspect of this tooth and associated with this was the presence of a foreign body (a fingernail fragment) that was removed (Figs 3-6). Following removal of the foreign body and gentle debridement of the pocket it was possible to express pus and inflammatory exudate from this site. From an orthodontic perspective he presented with a mild Class 3 malocclusion with severe crowding present in the upper arch associated with a buccally displaced upper left canine and an impacted upper right second premolar that remained unerupted and potentially excluded.

Radiographic examination confirmed a chronic apical area associated with the non-vital upper left lateral incisor, which communicated with the periodontal lesion on the mesial aspect of the canine (Fig. 7). A dental panoramic tomograph (DPT) confirmed the presence of all permanent teeth apart from the third molars. It also confirmed the presence of the impacted upper right second

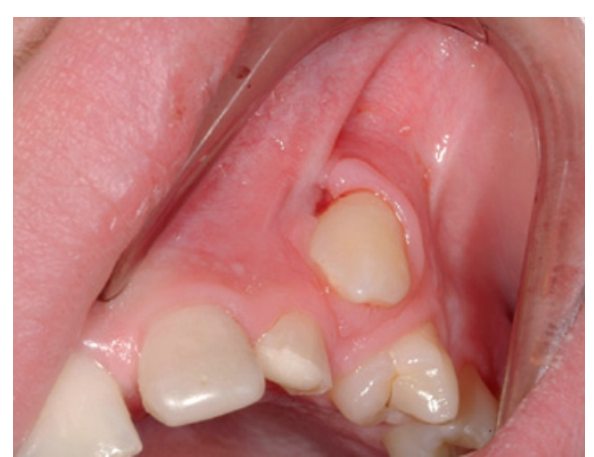

Fig. 1 Showing buccally placed canine with associated gingival inflammation

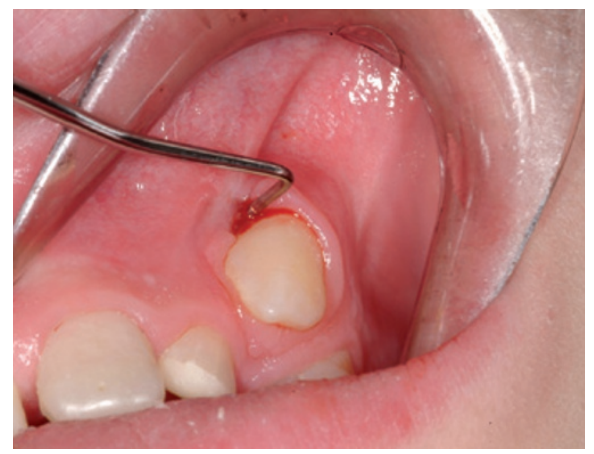

Fig. 2 Showing $8 \mathrm{~mm}$ periodontal pocket

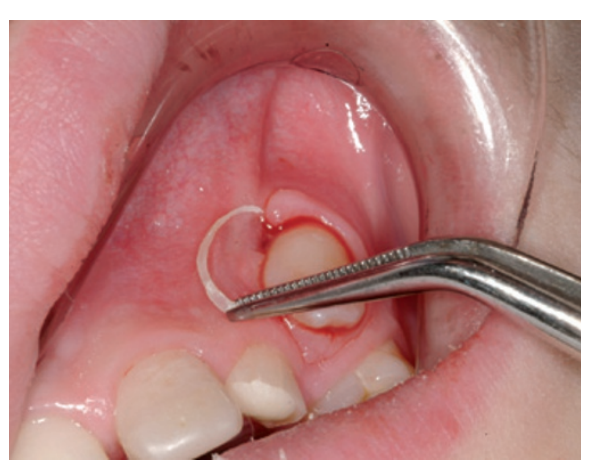

Fig. 3 Showing removal of fingernail fragment 
premolar that was potentially excluded from the arch and a late developing lower right second molar. A diagnosis was made of concurrent periodontal and endodontic lesion associated with the apical pathology related to the non-vital lateral and foreign body induced periodontal abscess affecting the canine.

The management involved restorative follow-up to carry out further debridement of the pocket on the mesial aspect of the upper left canine and to complete root canal therapy on the upper left lateral incisor. When his dental health was stable it was planned to arrange an orthodontic review and plan appliance treatment with extractions to be confirmed at that stage.

Following initial restorative care, the patient repeatedly failed to return for the completion of his treatment including definitive root canal therapy and he has also failed to attend for subsequent orthodontic review.

\section{DISCUSSION}

There are few reports in the literature regarding subgingival foreign body embedment. A literary search of electronic databases returned 11 articles, 7 of which are relevant when the keywords 'subgingival foreign body' are searched for. These include a case involving an orthodontic separator that caused a periodontal abscess ${ }^{3}$ and another where a similar foreign body caused localised periodontitis. ${ }^{4}$

A report by Manakou et al. describes a case of foreign body embedment with longterm consequences in the form of gingival recession. ${ }^{5}$ There are at least two other reports in the literature involving fingernails. ${ }^{6}$ Although this is thought to be relatively uncommon, the most frequently reported oral habit is onychophagia (fingernail biting) and with this habit there is the potential for a fingernail becoming lodged in the oral tissues. $^{2}$

Other previously isolated foreign bodies have included:

- Pins $^{7,8}$

- Staples ${ }^{9}$

- A metal ring ${ }^{10}$

- A gold chain ${ }^{10}$

- Wood $^{11}$

- Food items, such as nuts and seeds ${ }^{5}$

- Jewellery ${ }^{5}$

- Screws ${ }^{5}$

- Magnets ${ }^{5}$

- Plastic tubing. ${ }^{5}$

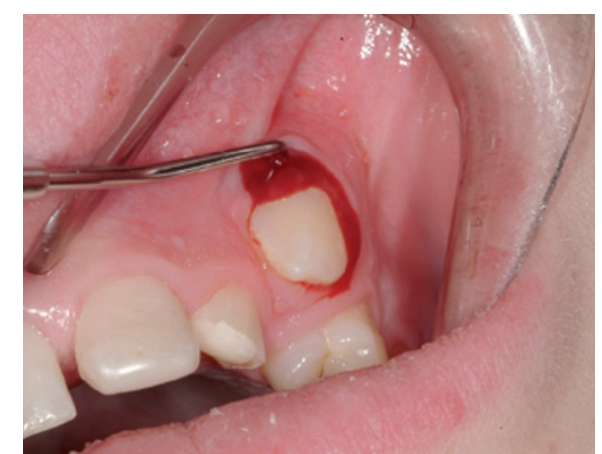

Fig. 4 Showing associated periodontal pocket after removal of fingernail fragment

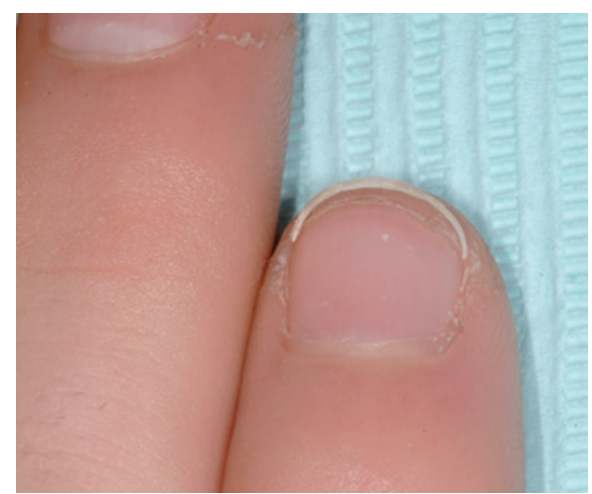

Fig. 5 Fingernail fragment pictured with offending fingernail

The current case demonstrates foreign body embedment but the diagnosis is complicated by the presence of a concurrent endodontic lesion on the adjacent lateral incisor. The radiographic image (Fig. 7) confirms the presence of an extensive apical area extending on to the mesial aspect of the canine, which is consistent with the clinical history of trauma and loss of vitality of the lateral incisor and subsequent chronic periapical infection. The radiograph, however, did not reveal the presence of a foreign body and this was only detected following careful clinical examination. Failure to detect the presence of the foreign body would have resulted in persistence of the periodontal infection and likely persistence of the concurrent periapical infection on the lateral incisor.

\section{CONCLUSION}

This case underlines the importance of taking a comprehensive history combined with thorough clinical assessment. Although foreign body impactions are relatively rare, they should be considered in a differential diagnosis, especially when the clinician is presented with an unusual clinical picture. ${ }^{1}$

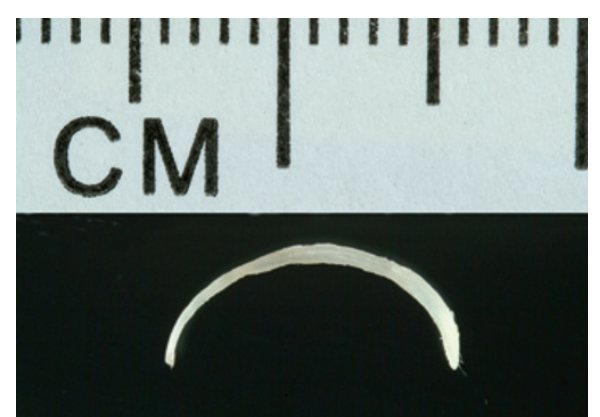

Fig. 6 Fingernail fragment pictured with ruler to show size
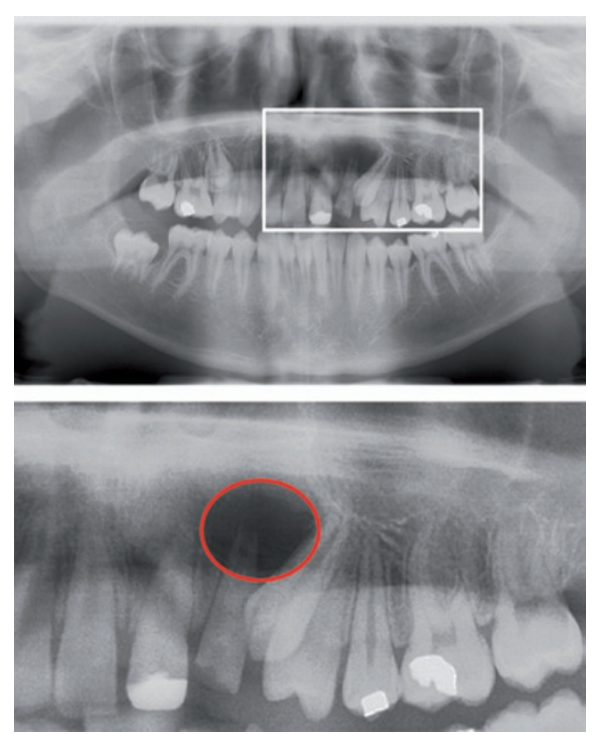

Fig. 7 A panoramic radiograph showing associated periapical radiolucency

1. Creath C J, Steinmetz S, Roebuck R. Case report. Gingival swelling due to a fingernail biting habit. J Am Dent Assoc 1995; 126: 1019-1021

2. Gale S, Hall E, Bhujel N, McKaig S. Common Things are common: a case series of oral foreign bodies in paediatric patients. Dent Update 2009; 36: 53-58

3. Becker T, Neronov A. Orthodontic elastic separatorinduced periodontal abscess: a case report.

Case Rep Dent 2012; 463903

4. Harrington Z, Darbar U. Localised periodontitis associated with an ectopic orthodontic separator. Prim Dent Care 2007; 14: 5-6.

5. Manakou A, Kavvadia K, Silvestros S, Angelopoulou E. Subgingival foreign body embedment in a preschool child: management with three and a half years follow-up. Eur J Paediatr Dent 2004; 5: 46-49.

6. Krejci C B. Self-inflicted gingival injury due to habitual fingernail biting. J Periodonto/ 2000; 71: 1029-1031.

7. Srivastava N, Vineeta N. Foreign body in the periradicular area. J Endod 2001; 27: 593-594.

8. Turner $\mathrm{CH}$. An unusual foreign body. Oral Surg Oral Med Oral Pathol 1983; 56: 226.

9. McAuliffe N, Drange N A, Hunter B. Staple diet: a foreign body in a tooth. Int J Paediatr Dent 2005; 15: 468-471.

10 .Colaneri L, Granger T R. Gold chain found between maxillary incisors. Oral Surg Oral Med Oral Pathol 1982; 54: 701.

11. Soubhia A M, Riberio A C, Martins L D, Silva A R, Lopes M A. Unusual wooden foreign body in the palate. Br Dent J 2007; 203: 573-574. 